\title{
Functional Interaction between Amyloid- $\beta$ Precursor Protein and Peripherin Neurofilaments: A Shared Pathway Leading to Alzheimer's Disease and Amyotrophic Lateral Sclerosis?
}

\author{
Virgil Muresan Christine Villegas Zoia Ladescu Muresan
}

Department of Pharmacology and Physiology, Rutgers, The State University of New Jersey, New Jersey Medical School, Newark, N.J., USA

\section{Key Words}

Alzheimer's disease - Amyotrophic lateral sclerosis . Amyloid- $\beta$ precursor protein $\cdot$ Intraneuronal amyloid- $\beta$ peptide $\cdot$ Neurofilaments $\cdot$ Peripherin $\cdot$ Mechanism of neurodegenerative diseases · Motor neurons · Disease crosstalk

\begin{abstract}
Background and Objective: The pathology of amyotrophic lateral sclerosis (ALS), a neurodegenerative disorder affecting motor neurons, comprises aberrant accumulations of neurofilaments; mutations in the peripherin subunit of neurofilaments have been identified in some forms of ALS. Recently, the amyloid- $\beta$ precursor protein (APP), a key element for the pathology of Alzheimer's disease (AD), was linked to ALS. Here, we provide evidence that the generation of the $\mathrm{N}$-terminal fragment of APP, SAPP, relies on peripherin neurofilaments. This finding could relate to a novel molecular mechanism dysregulated in ALS and/or AD. Methods and Results: The production and the fate of SAPP were studied with the brainstem-derived, neuronal cell line, CAD, which expresses endogenous peripherin. We show that SAPP and C-terminal fragments (CTF) are generated to a large extent in the neuronal soma. We find that SAPP, but not CTF, associates with filamentous structures that delineate the nuclear
\end{abstract}

lamina, extend to the cell periphery and immunostain for peripherin. The depletion of peripherin with siRNA eliminates the filamentous immunostaining of SAPP. Conclusion: Our results indicate that a fraction of APP is cleaved by $\beta$-secretase in the soma and that the generated SAPP becomes associated with perinuclear peripherin neurofilaments. These findings link the metabolism of APP - which is dysregulated in $A D$ - to the organization of neurofilaments - which is abnormal in ALS - and suggest a possible crosstalk/overlap between the molecular mechanisms of these diseases.

Copyright $\odot 2013$ S. Karger AG, Basel

\section{Background and Objective}

Most neurodegenerative diseases, including Alzheimer's disease (AD) and amyotrophic lateral sclerosis (ALS), have currently no cure. This situation is largely explained by our lack of sufficient knowledge about the mechanisms underlying the pathogenesis of these diseases. Neuronal degeneration and death, possibly linked to altered trafficking and metabolism of amyloid- $\beta$ precursor protein (APP) leading to the generation of potentially toxic APP fragments, is considered a probable cause of the profound memory deficits characteristic to $\mathrm{AD}[1,2]$. In ALS,

\section{KARGER}

E-Mail karger@karger.com

www.karger.com/ndd
(C) 2013 S. Karger AG, Basel

$1660-2854 / 13 / 0133-0122 \$ 38.00 / 0$ 


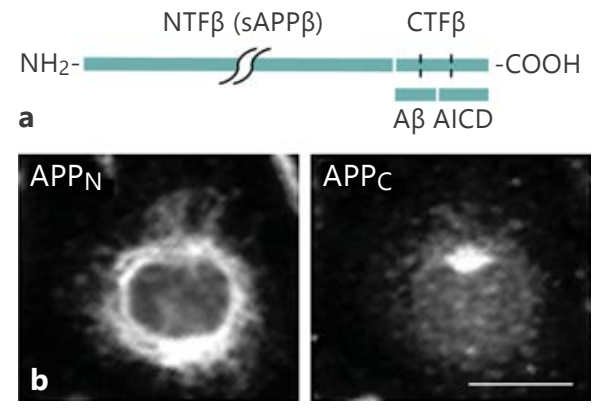

Fig. 1. Segregated distribution of APP-derived polypeptides. a Diagram showing the polypeptide fragments resulting from the cleavage of APP by $\beta$ - and $\gamma$-secretase. Cleavage by $\beta$-secretase produces NTF $\beta$ (also referred to as SAPP $\beta$ ) and CTF $\beta$. A $\beta$ and the APP intracellular domain AICD are obtained from CTF $\beta$ by cleavage by $\gamma$-secretase. The dotted lines mark the transmembrane domain. b Antibodies recognizing the $\mathrm{N}-\left(\mathrm{APP}_{\mathrm{N}}\right)$, but not C-terminal $\left(\mathrm{APP}_{\mathrm{C}}\right)$ epitopes of APP label a filamentous, perinuclear compartment in the soma of CAD neuronal cells. Scale bar $=20 \mu \mathrm{m}$.

upper and lower motor neurons are progressively lost by poorly understood mechanisms that, among others, lead to the disruption of the neurofilament networks and - ultimately - denervation of neuromuscular junctions $[3,4]$. A recent study proposed that APP actively contributes to the neuronal pathology in certain forms of ALS [5]. Here, we provide evidence that the proteolytic processing of APP, and intraneuronal localization of APP-derived fragments depend on intact peripherin-containing neurofilaments present in the soma. These results point to a possible crosstalk between the molecular machineries involved in the pathogenesis of AD and ALS.

\section{Methods}

In this study, we employed CAD neuronal cells [6-8], a brainstem-derived, neuronal cell line that, similar to the motor neurons, expresses peripherin. CAD neuronal cells also express APP, which is processed by secretases to generate the characteristic proteolytic fragments that are relevant to $\mathrm{AD}: \mathrm{sAPP} \beta, \mathrm{CTF} \beta, \mathrm{A} \beta$ and $\mathrm{AICD}$ [9-11] (fig. 1a). Immunocytochemistry, immunoblotting and transfection with peripherin siRNA (Santa Cruz Biotechnology) plus green fluorescent protein (GFP; to visualize transfected cells) were carried out as described $[12,13]$. The antibodies recognizing epitopes from the N- (22C11) or C-terminal region (AB5352) of APP were from Millipore. Previous work indicated that in CAD neuronal cells, the antibody 22C11 largely detects sAPP (N-terminal fragment of APP) rather than full-length APP [9]. The antiperipherin antibody was kindly provided by Dr. Robert Goldman (Northwestern University).

Functional Interaction between APP and Peripherin Neurofilaments

\section{Results}

Our previous studies with cultured neurons and mouse brain in situ have shown that a significant fraction of APP is proteolytically cleaved by secretases in the neuronal soma, leading to the generation of $\mathrm{N}$ - and C-terminal fragments (sAPP and CTF; fig. 1a) that are targeted to distinct neuronal compartments [9]. Immunocytochemistry with antibodies that detect epitopes within the $\mathrm{N}$ - and C-terminal regions of APP showed largely nonoverlapping distributions, with the antibodies to $\mathrm{N}$ - but not $\mathrm{C}$-terminal epitopes labeling a network of tubular structures surrounding the nucleus (fig. 1b). These results indicate that in these conditions, the antibodies to N-terminal epitopes largely reveal sAPP, not full-length APP. The immunolabeling pattern of sAPP was reminiscent of the cytoskeletal neurofilament network, which is tightly associated with the nuclear envelope. With dual immunolabeling, we showed that the SAPP detected in the soma displays strong colocalization with peripherin (fig. 2), a subunit of neurofilaments expressed in the adult neurons of the peripheral nervous system and the brainstem [14]. We note that colocalization of APP with cytoskeletal filaments that are not microtubules was previously detected in glial cells [15].

To examine the link between the distribution of sAPP and that of neurofilaments, we tested whether the depletion of peripherin protein affects the distribution of sAPP. Although neurofilament proteins are long lived, CAD neuronal cells showed significantly diminished peripherin levels 4 days after transfection with peripherin siRNA (fig. 3). Cells with reduced peripherin levels, visualized by the cotransfected GFP, lacked perinuclear peripherin and no longer showed the filamentous, perinuclear distribution of sAPP evident in nontransfected cells (fig. 3). This result indicates that the presence of neurofilaments is required for either the generation of sAPP from full-length APP, or for the localization of sAPP-containing membrane vesicles along neurofilaments. Thus, this work uncovers a functional interaction between APP and peripherin neurofilaments in CAD neuronal cells.

\section{Conclusions}

This study identifies the neurofilament protein, peripherin, as a potential regulator of APP metabolism and trafficking in neurons. One possibility is that the proteolytic processing of APP occurs in a compartment - endosomes, or part of the endoplasmic reticulum [16] - associated with the neurofilament cytoskeleton. Alternative- 

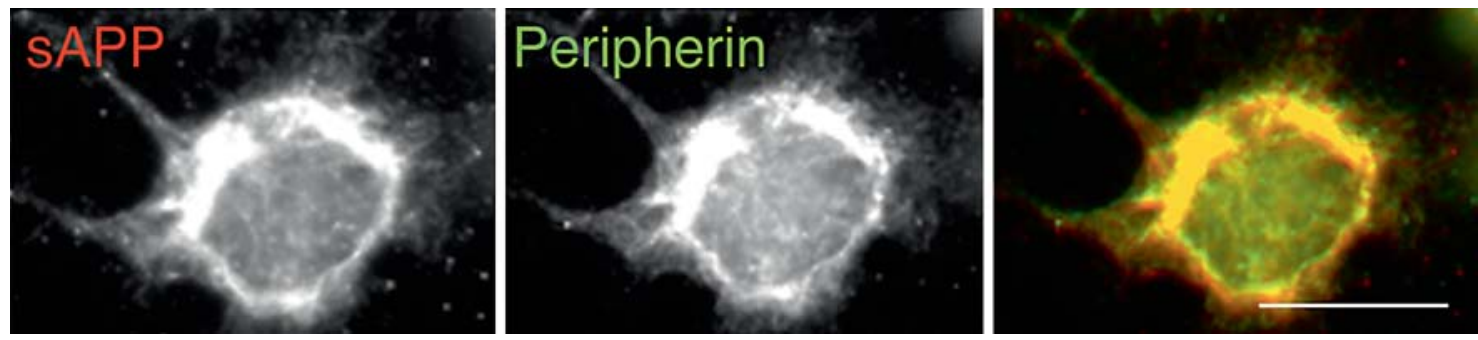

Fig. 2. The APP N-terminal epitopes detected with antibody $22 \mathrm{C} 11$ (likely sAPP) colocalize with the peripherinpositive neurofilaments in a region surrounding the nucleus of CAD neuronal cells. Scale bar $=20 \mu \mathrm{m}$.

Fig. 3. Silencing the expression of prph by transfection of CAD neuronal cells with peripherin siRNA diminishes the expression of peripherin protein by over $50 \%$ (immunoblot, top right; the Ponceau-Sstained transfers at the bottom right show comparable loads in siRNA-treated and control cells) and abrogates the characteristic, perinuclear localization of both peripherin and sAPP (immunofluorescence images). Cotransfected GFP marks the siRNA-treated cells (left and middle images). A nontransfected, control cell is shown for comparison (right images). For increased resolution of sAPP labeling, the top images are shown in black and white. Scale bars $=20 \mu \mathrm{m}$. Molecular weight markers are given in kilodaltons.

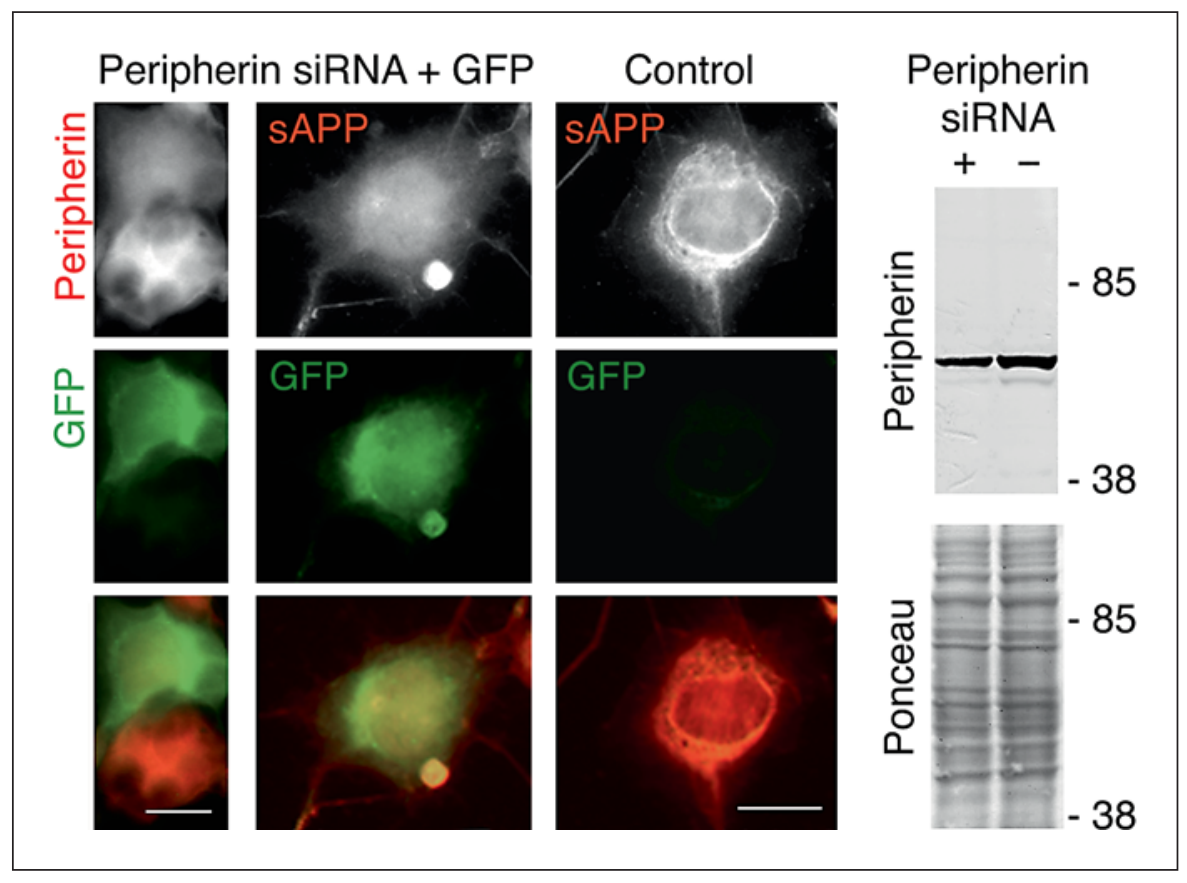

ly, the neurofilaments could play a role in the segregation of the sAPP from CTF, once they have been generated in a somatic compartment, by selectively anchoring, and concentrating, SAPP-containing vesicles in the perinuclear region. This region has been recently shown to be a site for accumulation of APP-derived polypeptides, with relevance to $\mathrm{AD}$ [17]. Interestingly, ALS-specific mutations in the peripherin gene $[18,19]$ lead to abnormal neurofilament organization [for a review, see ref. 20]. In principle, these cytoskeletal changes could cause abnormal processing - and thus altered function - of APP, a possibility supported by our results. We propose that this functional interaction between APP and peripherin neurofilaments may provide the basis for crosstalk between the molecular mechanisms of disease in AD and ALS.

\section{Acknowledgments}

This work was supported by the National Institutes of Health award AG039668 (to Z.M.) and New Jersey Health Foundation grants (to Z.M. and V.M.).

References

- 1 Muresan V, Muresan Z: Is abnormal axonal transport a cause, a contributing factor or a consequence of the neuronal pathology in $\mathrm{Al}$ zheimer's disease? Future Neurol 2009;4:761773.

2 Zheng H, Koo EH: Biology and pathophysiology of the amyloid precursor protein. Mol Neurodegener 2011;6:27.

- 3 Wijesekera LC, Leigh PN: Amyotrophic lateral sclerosis. Orphanet J Rare Dis 2009; $4: 3$. 
4 Xiao S, McLean J, Robertson J: Neuronal intermediate filaments and ALS: a new look at an old question. Biochim Biophys Acta 2006; 1762:1001-1012.

$\checkmark 5$ Bryson JB, Hobbs C, Parsons MJ, Bosch KD, Pandraud A, Walsh FS, Doherty P, Greensmith L: Amyloid precursor protein (APP) contributes to pathology in the SOD1(G93A) mouse model of amyotrophic lateral sclerosis. Hum Mol Genet 2012;21:3871-3882.

-6 Muresan Z, Muresan V: Seeding neuritic plaques from the distance: a possible role for brainstem neurons in the development of Alzheimer's disease pathology. Neurodegener Dis 2008;5:250-253.

7 Muresan Z, Muresan V: CAD cells are a useful model for studies of APP cell biology and Alzheimer's disease pathology, including accumulation of $\mathrm{A} \beta$ within neurites. SWAN Alzheimer Knowledge Base. Alzheimer Research Forum, Boston, 2009.

8 Qi Y, Wang JK, McMillian M, Chikaraishi DM: Characterization of a CNS cell line, CAD, in which morphological differentiation is initiated by serum deprivation. J Neurosci 1997; 17:1217-1225.

-9 Muresan V, Varvel NH, Lamb BT, Muresan Z: The cleavage products of amyloid- $\beta$ precursor protein are sorted to distinct carrier vesicles that are independently transported within neurites. J Neurosci 2009;29:3565-3578.
10 Muresan Z, Muresan V: A phosphorylated, carboxy-terminal fragment of $\beta$-amyloid precursor protein localizes to the splicing factor compartment. Hum Mol Genet 2004;13:475488.

11 Muresan Z, Muresan V: Neuritic deposits of amyloid- $\beta$ peptide in a subpopulation of central nervous system-derived neuronal cells. Mol Cell Biol 2006;26:4982-4997.

12 Muresan Z, Muresan V: c-Jun $\mathrm{NH}_{2}$-terminal kinase-interacting protein-3 facilitates phosphorylation and controls localization of amyloid- $\beta$ precursor protein. J Neurosci 2005;25:3741-3751.

13 Muresan Z, Muresan V: Coordinated transport of phosphorylated amyloid- $\beta$ precursor protein and c-Jun $\mathrm{NH}_{2}$-terminal kinase-interacting protein-1. J Cell Biol 2005;171:615625.

14 Yuan A, Sasaki T, Kumar A, Peterhoff CM, Rao MV, Liem RK, Julien JP, Nixon RA: Peripherin is a subunit of peripheral nerve neurofilaments: implications for differential vulnerability of CNS and peripheral nervous system axons. J Neurosci 2012;32:8501-8508.
5 Berkenbosch F, Refolo LM, Friedrich VL Jr, Casper D, Blum M, Robakis NK: The Alzheimer's amyloid precursor protein is produced by type I astrocytes in primary cultures of rat neuroglia. J Neurosci Res 1990;25:431440.

16 Muresan V, Muresan Z: A persistent stress response to impeded axonal transport leads to accumulation of amyloid- $\beta$ in the endoplasmic reticulum, and is a probable cause of sporadic Alzheimer's disease. Neurodegener Dis 2012;10:60-63.

17 Glabe C: Conformational diversity of amyloid in human AD brain. The 11th International Conference on Alzheimer's and Parkinson's Disease, Florence, Italy, March 6-10, 2013.

18 Corrado L, Carlomagno Y, Falasco L, Mellone S, Godi M, Cova E, Cereda C, Testa L, Mazzini L, D’Alfonso S: A novel peripherin gene (PRPH) mutation identified in one sporadic amyotrophic lateral sclerosis patient. Neurobiol Aging 2011;32:552, e551-e556.

19 Leung CL, He CZ, Kaufmann P, Chin SS, Naini A, Liem RK, Mitsumoto H, Hays AP: A pathogenic peripherin gene mutation in a patient with amyotrophic lateral sclerosis. Brain Pathol 2004; 14:290-296.

20 Millecamps S, Julien JP: Axonal transport deficits and neurodegenerative diseases. Nat Rev Neurosci 2013;14:161-176. 